\title{
De la pertinence de l'étude des TICE par les SHS
}

\section{Cathia Papi}

\section{OpenEdition}

\section{Journals}

Édition électronique

URL : http://journals.openedition.org/communicationorganisation/4209

DOI : 10.4000/communicationorganisation.4209

ISSN : 1775-3546

\section{Éditeur}

Presses universitaires de Bordeaux

\section{Édition imprimée}

Date de publication : 1 juin 2013

Pagination : 183-192

ISBN : 978-2-86781-883-7

ISSN : 1168-5549

\section{Référence électronique}

Cathia Papi, « De la pertinence de l'étude des TICE par les SHS », Communication et organisation [En ligne], 43 | 2013, mis en ligne le 01 juin 2015, consulté le 19 avril 2019. URL : http:// journals.openedition.org/communicationorganisation/4209; DOI : 10.4000/ communicationorganisation.4209 


\title{
De la pertinence de l'étude des TICE par les SHS
}

\author{
Cothio Papi ${ }^{1}$
}

«SABOTAGE - Un élève réécrit une page Wikipédia pour ne pas être accusé de plagiat ». Tel est l'un des titres affiché sur le « Big browser» du site Le Monde. fr du 17 avril 2012. Cette histoire d'élève ayant fait son devoir de français sur Le Meilleur des mondes de Huxley en faisant du copier-coller de Wikipédia, puis en changeant la page du site en question afin que sa professeure ne découvre pas la supercherie, a suscité 69 commentaires en trois heures, signe que les usages des technologies ou ressources médiatisées n'en finissent pas de surprendre. Alors que l'encyclopédie a pour vocation originelle le recueil et la diffusion des savoirs, un tel usage de Wikipédia inverse effectivement la logique de construction collective visant l'enrichissement des savoirs en logique d'enrichissement personnel (tout du moins en termes de note escomptée), impliquant la destruction individuelle des savoirs collectifs.

Depuis les années 1980, un grand nombre de recherches pointent l'existence de décalages entre les usages anticipés par les concepteurs et ceux mis en œuvre par les usagers. Ainsi, les études portant sur les usages du Minitel ont mis en relief que l'usage initialement anticipé de consultation des bases de données depuis chez soi était surpassé par l'usage de la messagerie ou des espaces ludiques. Plus récemment, des observations du même acabit ont été réalisées concernant les SMS (Short Message Service) dont la vocation de transmission de messages des opérateurs aux usagers a rapidement laissé place à des pratiques de communication amenant avec elles le développement d'un écrit oralisé particulier.

Si le questionnement des usages sociaux généralement « définis comme les patterns d'usages individuels ou collectifs (strates, classes) qui s'avèrent relativement stabilisés sur une période historique, à l'échelle d'ensembles sociaux plus larges (communautés, sociétés, civilisations)» (Jauréguiberry et Proulx, $2011: 25)$ a d'abord concerné la sphère des usages domestiques, il

1 Cathia Papi est Maître de Conférences en Sciences de l'Information et de la Communication à l'Université de Picardie Jules Verne où elle est en charge du C2i. Ses recherches sur les usages des TIC et la formation à distance des adultes s'inscrivent dans le cadre de l'équipe COMETIC (communication éducation et TIC) du CURAPP UMR 7319 ; Cathia.papi@u-picardie.fr 
tend de plus en plus à interroger le champ de l'éducation et de la formation. Ainsi, bon nombre de travaux sur les technologies de l'information et de la communication dans l'éducation (TICE) soulignent, également, les écarts existants entre attentes des concepteurs de dispositifs et pratiques de formation, tout comme entre attentes gouvernementales et systèmes scolaires ou pratiques enseignantes (Papi, 2009 ; Albero et Thibault, 2009 ; Chaptal, 2007 ; etc.). Cependant, alors que ce constat d'un décalage entre usages prévus et usages effectifs semble éloigné de la conception et donc de l'action sur les EIAH (environnements informatiques pour l'apprentissage humain), dans quelle mesure la recherche en SHS (sciences humaines et sociales) est-elle pertinente pour l'étude des TICE ?

\section{Ancrage scientifique}

Outre les travaux centrés sur les dimensions éthiques, philosophiques ou épistémologiques des TICE, deux orientations principales sont repérables dans les recherches actuelles : l'une, issue des travaux du milieu du $X^{\text {ème }}$ siècle sur la cybernétique et l'intelligence artificielle s'intéresse davantage à la conception des EIAH ; l'autre privilégie l'étude des usages des innovations techniques et des changements qui les accompagnent dans différentes sphères d'activité.

Si l'intérêt pour les TICE est plus récent dans les recherches de sciences humaines et sociales que dans les disciplines informatiques, il s'est notamment accru avec le développement des sciences de l'éducation et des sciences de l'information et de la communication s'intéressant aux innovations technologiques et pédagogiques et, de façon plus générale, aux usages des TIC dans l'éducation et la société.

Loin du déterminisme technique, les recherches s'intéressant au rapport homme-technologie en termes d'appropriation font ainsi ressortir l'autonomie des pratiques instrumentées. Au centre de cette approche, l'ouvrage L'invention du quotidien de M. de Certeau (1980) pointe les stratégies, ruses et bricolages c'est-à-dire la créativité de l'homme dans son rapport à la machine. De même, J. Perriault (1989) voit dans le décalage entre usages prévus et usages observés, la marque de l'acteur ou, plus précisément celle de sa logique d'action. Cette approche trouve ses prolongements actuels, d'une part, dans une sociologie des usages prenant davantage en compte le contexte dans lequel se situe l'usage et, d'autre part, dans une sociologie des non-usages, cherchant à dépasser les notions d'inégalités ou fractures numériques, souvent liées à des questions d'équipement, pour comprendre les rationalités (Von Pape et Martin, 2010) ou logiques de non-usages situées (Papi, à paraître). Ainsi, selon S. Wyatt $(2010: 34)$ : «Le non-usage n'est pas la seule pratique à déchiffrer. De façon symétrique, l'usage se doit également d'être explicité et ne devrait pas être considéré comme une pratique normale et tenue pour acquise. [...] les choix 
des individus sont enracinés dans l'économie morale du ménage, dans les relations sociales et culturelles ainsi que dans les relations de production. »

Dans le champ de la formation à distance, la question des usages porte notamment sur les moyens de communication proposés et amène souvent à constater un échange d'informations là où est attendue une construction collaborative de connaissances et, de façon plus générale, une moindre participation en dehors des interactions entrant dans le cadre d'une évaluation (Papi, 2009 ; Caviale et Bruillard, 2009). Cependant, l'évaluation n'est certainement pas le seul motif des usages repérés dans les pratiques d'apprentissage. Dès lors, de quel ordre de facteurs relèvent les freins à l'usage des TICE dans le cadre de la formation à distance ? Autrement dit, s'agitil de repenser la conception du dispositif au niveau technique ou au niveau pédagogique voire social?

Nous proposons d'ouvrir des pistes de réflexion en nous intéressant à un dispositif hybride consacré à la préparation du C2i.

\section{Approche empirique}

Les différentes recherches menées jusqu'alors sur les non-usages des TIC ont mis en avant que certaines caractéristiques sociodémographiques et socioéconomiques expliquent davantage les fréquences ou types d'usages que d'autres. Ainsi, la plupart des enquêtes sur les (non-) usages des TIC, qu'il s'agisse de connexion Internet (Granjon, 2009 ; Boutet et al., 2009 ; Smith, 2010 ; Wyatt, 2010) ou de téléphones portables (Von Pape et al., 2010) mettent systématiquement en évidence que les jeunes, et notamment ceux ayant suivi un enseignement supérieur, sont les mieux équipés. Afin de mettre de côté la question de l'équipement, nous proposons donc de nous intéresser à une population d'usagers à la fois jeunes et liée à l'enseignement supérieur : les étudiants.

Alors que les traces laissées sur la plate-forme de formation par l'envoi de messages ou la consultation des cours permettent de repérer certains usages mais sans en fournir d'explication, nous avons interrogé les étudiants participant au dispositif de préparation du C2i à l'Université de Picardie Jules Verne (UPJV) en leur soumettant un questionnaire au terme de leur formation. Tandis qu'une variété de ressources leur est proposées - cours PDF accessible en ligne et téléchargeable, cours sous forme de diaporama interactif ou de screencasts, e-mail, forums et chats pour communiquer avec le moniteur aussi bien qu'avec les étudiants - quels sont les usages et nonusages rencontrés et comment les expliquer?

L'analyse via des tris à plat et croisés des réponses fournies en 2011-2012 par 1365 étudiants (soit plus de la moitié des inscrits) ainsi que par 57 moniteurs dans le cadre de leur bilan d'activité permet d'avancer quelques pistes de réflexion. 


\section{Premiers constats}

Dans le cadre de la préparation au C2i sur la plate-forme INES intégrée dans l'environnement numérique de travail (ENT) de l'UPJV, les étudiants ont à réaliser cinq activités et à répondre à un $\mathrm{QCM}$ de 45 questions afin de permettre la validation des différents domaines de compétences du référentiel. Pour ce faire, les étudiants (majoritairement inscrits en L1), réunis en groupes d'au maximum 25, sont encadrés par des moniteurs C2i, c'est-à-dire des étudiants généralement inscrits en master, ayant obtenu leur $\mathrm{C} 2 \mathrm{i}$ et suivi une formation au monitorat. Les étudiants suivent deux séances de TD avec leur moniteur puis continuent leur formation en ligne avec l'accompagnement de ce dernier. Ils doivent ainsi réaliser cinq activités (quatre sont individuelles, une est collaborative) à distance avant de revenir en salle de classe pour un TD de préparation au QCM puis le passage du QCM en ligne, sous la surveillance de leur moniteur ${ }^{2}$. À l'issue de leur formation, $30 \%$ des étudiants déclarent que les activités étaient difficiles voire très difficiles. Seulement $9 \%$ trouvent les activités faciles voire très faciles, pour $60 \%$ elles sont faisables. De même, pour $69 \%$ des étudiants le QCM est difficile voire très difficile, pour $35 \%$ faisable et pour $6 \%$ facile voire très facile. Ces déclarations rejoignent celles posées en termes d'apprentissage en ce sens où $9 \%$ disent ne rien avoir appris en préparant le $\mathrm{C} 2 \mathrm{i}, 44 \%$ pensent avoir appris peu de choses tandis pour $47 \%$ la préparation du C2i fut l'occasion de nombreux apprentissages, voire de l'ensemble de leurs connaissances dans le domaine.

Les analyses de Khi2 mettent en relief le lien existant entre le degré de difficulté ressenti relativement aux activités et au QCM (Khi2 $=224,5$ ddl=4 $\mathrm{p}=0,001$ ), d'une part ainsi que le lien entre ce degré de difficulté et le sentiment d'apprentissage (Khi2=92,7 ddl=4 p=0,001). Ce dernier paraît lui-même lié de façon très significative (analyse des Khi2 avec $d d l=6, p=0,001$ ) à l'usage des ressources aussi bien en termes de communication (interactions avec les étudiants : Khi2=29.1 ; interactions avec les moniteurs : Khi2=28,7) qu'en termes de consultation des cours (PDF en ligne : Khi2=79,7 ; PDF hors ligne : Khi2=55,6; diapo : Khi2=45,1) exception faite des screencasts. Enfin, le lien est également très significatif (Khi2 avec ddl $=9 \mathrm{p}=0,001)$ entre le fait de consulter les cours au format PDF $($ Khi2=637,0), diapo (Khi2=474,0) ou vidéo $(\mathrm{Khi} 2=306,9)$. Autrement dit, plus les étudiants rencontrent des difficultés dans la préparation du C2i, plus ils mobilisent l'ensemble des ressources à leur disposition et plus ils ont le sentiment d'avoir appris et inversement, rien de surprenant jusque là. Il est par contre plus étonnant de noter que parmi les 54,8\% étudiants n'ayant jamais ou très peu communiqué avec leur moniteur seulement $5 \%$ considéraient les activités comme faciles ou très faciles; de même parmi les $43,7 \%$ n'ayant jamais ou très peu communiqué

2 Pour faciliter la lecture, nous parlons d'étudiant et de moniteur au masculin mais il convient de ne pas oublier que près des deux tiers des étudiants ayant répondu au questionnaire sont des étudiantes et qu'un peu plus de la moitié des moniteurs sont des monitrices. 
avec les étudiants, seuls 2,5\% trouvaient les activités faciles voire très faciles ; il en va de même pour 5,6 \% des 39,5\% ayant peu voire pas regardé les cours $\mathrm{PDF}$ en ligne ainsi que pour 7,9\% des 70,2\% ne s'étant pas ou peu servi des diaporamas ou pour $8,1 \%$ des $78,4 \%$ n'ayant pas ou brièvement consultés les vidéos de formation ainsi que le détaille le tableau 1.

\begin{tabular}{|c|c|c|c|c|c|}
\hline Pdf en ligne/Activités & $\begin{array}{c}\text { (Très) } \\
\text { Difficile }\end{array}$ & Faisables & $\begin{array}{c}\text { (Très) } \\
\text { Faciles }\end{array}$ & Total & $\begin{array}{c}\text { Khi2 }=29,9 \text { ddl }=6 \quad p=0,001 \\
\text { (Très significatif) }\end{array}$ \\
\hline Pas regardés & 5,2 & 7,4 & 2,6 & 15,2 & 39,5 \\
\hline Bref aperçu & 6,4 & 14,9 & 3,0 & 24,3 & \\
\hline Consultation de certains & 13,6 & 27,3 & 3,3 & 44,2 & \\
\hline Tous consultés & 5,3 & 10,1 & 0,9 & 16,3 & 00,0 \\
\hline Pdf hors ligne/Activités & $\begin{array}{l}\text { (Très) } \\
\text { Difficile }\end{array}$ & Faisables & $\begin{array}{l}\text { (Très) } \\
\text { Faciles }\end{array}$ & Total & $\begin{array}{c}\text { Khi2 }=10,7 \text { ddl }=6 \quad p=0,098 \\
\text { (Assez significatif) }\end{array}$ \\
\hline Pas regardés & 8,6 & 16,6 & 3,8 & 28,9 & \\
\hline Bref aperçu & 7,5 & 14,5 & 2,2 & 24,2 & 33,1 \\
\hline Consultation de certains & 9,9 & 20,5 & 3,2 & 33,6 & 46,9 \\
\hline Tous consultés & 4,5 & 8,1 & 0,7 & 13,3 & \\
\hline Diapo/Activités & $\begin{array}{l}\text { (Très) } \\
\text { Difficile }\end{array}$ & Faisables & $\begin{array}{l}\text { (Très) } \\
\text { Faciles }\end{array}$ & Total & $\begin{array}{c}\text { Khi2 }=18,9 \text { ddl }=6 \quad p=0,004 \\
\text { (Très significatif) }\end{array}$ \\
\hline Pas regardés & 11,9 & 23,9 & 5,6 & 41,3 & \\
\hline Bref aperçu & 8,6 & 17,9 & 2,3 & 28,9 & 70,2 \\
\hline Consultation de certains & 7,6 & 14,9 & 1,7 & 24,2 & 29,8 \\
\hline Tous consultés & 2,3 & 3,0 & 0,3 & 5,6 & \\
\hline Vidéos/Activités & $\begin{array}{c}\text { (Très) } \\
\text { Difficile }\end{array}$ & Faisables & $\begin{array}{c}\text { (Très) } \\
\text { Faciles }\end{array}$ & Total & $\begin{array}{c}\text { Khi2 }=10,7 \text { ddl }=6 \quad p=0,097 \\
\text { (Assez significatif) }\end{array}$ \\
\hline Pas regardés & 16,8 & 35,8 & 6,8 & 59,4 & 78,4 \\
\hline Bref aperçu & 6,5 & 11,1 & 1,3 & 19,0 & \\
\hline Consultation de certains & 5,6 & 10,8 & 1,4 & 17,8 & \\
\hline Tous consultés & 1,5 & 2,0 & 0,3 & 3,8 & 21,6 \\
\hline Com.moniteur/Activités & $\begin{array}{c}\text { (Très) } \\
\text { Difficile }\end{array}$ & Faisables & $\begin{array}{c}\text { (Très) } \\
\text { Faciles }\end{array}$ & Total & $\begin{array}{c}\text { Khi2 }=16,8 \text { ddl }=6 \quad p=0,01 \\
\text { (Très significatif) }\end{array}$ \\
\hline Jamais & 8,4 & 12,2 & 1,6 & 22,2 & 54,8 \\
\hline Rarement & 9,7 & 19,5 & 3,4 & 32,6 & \\
\hline Parfois & 7,5 & 18,2 & 2,5 & 28,2 & 45,2 \\
\hline Souvent & 4,8 & 9,9 & 2,3 & 17,0 & \\
\hline Com. étudiants / Activités & $\begin{array}{l}\text { (Trés) } \\
\text { Difficile }\end{array}$ & Faisables & $\begin{array}{l}\text { (Trés) } \\
\text { Faciles }\end{array}$ & Total & $\begin{array}{c}\text { Khi2 }=5,26 \text { ddl }=6 \quad p=0,513 \\
\text { (Peu significatif) }\end{array}$ \\
\hline Jamais & 7,0 & 12,7 & 2,2 & 21,9 & 4377 \\
\hline Rarement & 6,5 & 12,9 & 2,3 & 21,8 & 43,1 \\
\hline Parfois & 8,6 & 19,5 & 2,5 & 30,6 & 56,3 \\
\hline Souvent & 8,4 & 14,6 & 2,8 & 25,7 & \\
\hline Total & 30,5 & 59,7 & 9,8 & 100,0 & \\
\hline
\end{tabular}

Tableau 1 : Usages des ressources de formation et perceptions du niveau de difficulté des activités $(\%)$

De fait, la part des étudiants ne faisant pas usage des ressources parce que leurs connaissances leur suffisent est moindre comme le révèle le tableau 2. 


\begin{tabular}{|r|r|r|}
\hline Motifs de non-usage & Cours (pdf ou diapo) & Screencast \\
\hline Rébarbatifs, trop long & $66,9 \%$ & $45,9 \%$ \\
\hline Pas intéressant & $2,5 \%$ & $4,5 \%$ \\
\hline Pas adapté (équipement/contenu) & $0,3 \%$ & $0,7 \%$ \\
\hline Problèmes techniques d'accès & $0,8 \%$ & $1,7 \%$ \\
\hline Aide extérieure & $2,7 \%$ & $2,8 \%$ \\
\hline Désaffection pour l'informatique etinternet & $0,5 \%$ & $0,5 \%$ \\
\hline Non connaissance de son existence & $3,7 \%$ & $12,8 \%$ \\
\hline Connaissances suffisantes & $5,9 \%$ & $6,8 \%$ \\
\hline Préférences pour les autres contenus proposés & $1,2 \%$ & $13,1 \%$ \\
\hline Autre & $15,6 \%$ & $11,3 \%$ \\
\hline Total & $100,0 \%$ & $100 \%$ \\
\hline
\end{tabular}

Tableau 2 : Motifs de non-usage des cours et vidéos de formation (\%)

C'est avant tout l'aspect rébarbatif voire la longueur des ressources qui incite à ne pas les utiliser, ce qui est compréhensible dans le cas des cours relativement longs mais étonnant en ce qui concerne les screencasts d'environ 3 minutes. Nous avons démontré par ailleurs (Papi, à paraître) que la préférence pour le cours au format PDF pouvait s'analyser en termes de conflits instrumentaux et s'expliquer par les habitudes d'apprentissage des étudiants. Force est de constater que la faiblesse des freins d'ordre technique tant en ce qui concerne l'accès aux cours et espaces de communication que la correspondance entre les systèmes et logiciels présentés dans les cours et ceux utilisés chez soi. Il convient donc de chercher ailleurs les phénomènes influençant les pratiques des étudiants.

\section{Éléments de compréhension}

Deux pistes nous semblent notamment pouvoir être explorées pour comprendre les usages et pratiques des étudiants : d'une part, celle du rapport entre leurs usages des TIC dans la vie quotidienne et leurs usages dans le cadre de la formation; d'autre part, celle de l'influence de leur moniteur dans le choix des supports de formation.

La mobilisation des ressources proposées aux étudiants dans le cadre de la préparation du $\mathrm{C} 2 \mathrm{i}$ dépend plus largement des pratiques quotidiennes des étudiants comme l'illustre le tableau suivant :

\begin{tabular}{|c|c|c|c|c|}
\hline $\begin{array}{l}\text { Tps hebdomadaire } \\
\begin{array}{l}\text { Consultation des } \\
\text { cours PDF en ligne }\end{array}\end{array}$ & $\begin{array}{l}\text { Peu voire pas } \\
\text { consultés }\end{array}$ & $\begin{array}{l}\text { Consultation } \\
\text { de certains } \\
\text { cours }\end{array}$ & $\begin{array}{l}\text { Consultation } \\
\text { de tous les } \\
\text { cours }\end{array}$ & Total \\
\hline $4 \mathrm{~h}$ et moins & 13,0 & 10,8 & 4,4 & 28,3 \\
\hline entre 5 et 9 h & 14,8 & 17,1 & 6,1 & 38,0 \\
\hline entre 10 et $14 \mathrm{~h}$ & 8,1 & 9,6 & 3,3 & 21,0 \\
\hline entre 15 et $19 \mathrm{~h}$ & 1,9 & 4,1 & 1,2 & 7,2 \\
\hline 20 h et plus & 1,8 & 2,5 & 1,3 & 5,6 \\
\hline Total & 39,6 & 44,2 & 16,3 & 100,0 \\
\hline
\end{tabular}

Tableau 3 : Lien entre le temps passé à travailler les cours et la consultation du cours en pdf du C2i (\%) Khi2 $=19,5$ ddl= $=8$ p=0,012 (Très significatif) 
La fréquence de communication avec les autres étudiants est également corrélée au temps hebdomadaire passé à communiquer de façon synchrone en ligne (Khi2 $=23,3 \mathrm{dd} 1=4 \mathrm{p}=0,001)$.

Ces deux exemples mettent en relief l'impact des pratiques quotidiennes sur les pratiques d'apprentissage. Ainsi, plus les étudiants consacrent de temps au travail de leurs cours et devoirs plus ils tendent à se servir des cours PDF du C2i. De même, plus ils ont l'habitude de communiquer en ligne, plus ils tendent à communiquer avec les autres étudiants (ainsi que les moniteurs) dans le cadre de la préparation du C2i. Il n'est dès lors pas étonnant que ce soient les mêmes étudiants qui utilisent les différents types de ressources. Ainsi, les étudiants les plus communicatifs cherchent l'interaction aussi bien avec les autres étudiants qu'avec leur moniteur comme l'illustre les données ci-dessous.

\begin{tabular}{|c|l|l|l|l|l|l|l|l|l|l|}
\hline $\begin{array}{r}\text { Com. avec } \\
\text { moniteurs }\end{array}$ & \multicolumn{3}{|c|}{ Jamais } & \multicolumn{2}{c|}{ Rarement } & \multicolumn{2}{c|}{ Parfois } & \multicolumn{2}{c|}{ Souvent } & \multicolumn{2}{c|}{ Total } \\
$\begin{array}{c}\text { Com. avec d'autres } \\
\text { étudiants }\end{array}$ & Eff. & $\%$ & Eff. & $\%$ & Eff. & $\%$ & Eff. & $\%$ & Eff. & $\%$ \\
\hline Jamais & 101 & 7,4 & 83 & 6,1 & 71 & 5,2 & 43 & 3,2 & 298 & 21,9 \\
\hline Rarement & 68 & 5,0 & 103 & 7,6 & 80 & 5,9 & 46 & 3,4 & 297 & 21,8 \\
\hline Parfois & 83 & 6,1 & 146 & 10,7 & 126 & 9,2 & 62 & 4,5 & 417 & 30,6 \\
\hline Souvent & 50 & 3,7 & 112 & 8,2 & 108 & 7,9 & 81 & 5,9 & 351 & 25,8 \\
\hline Total & 302 & 22,2 & 444 & 32,6 & 385 & 28,2 & 232 & 17,0 & 1363 & 100,0 \\
\hline
\end{tabular}

Tableau 4 : Tri croisé des fréquences de communication des étudiants $\mathrm{C} 2 \mathrm{i}$ Khi2 $=46,6 d d l=9 p=0,001$ (Très significatif)

Si la rencontre en face-à-face est le moyen de communication privilégié avec ses camarades de classe, c'est principalement l'échange de courriels qui permet la communication avec le moniteur suivi de loin par le forum puis le chat. Face aux questions des étudiants, ainsi principalement reçues par $e$-mail, les moniteurs tendent en premier lieu à suggérer aux étudiants de trouver la réponse sur internet.

Le graphique ci-après (fig. 1) invite ainsi à penser que les pratiques des étudiants sont également influencées par les réactions des moniteurs qui les conduisent notamment à chercher réponses à leurs questions en dehors des ressources proposées dans le cadre de la formation ou à privilégier le forum et le cours au format PDF relativement aux espaces d'échange synchrone et aux cours au format diapo ou vidéo. 


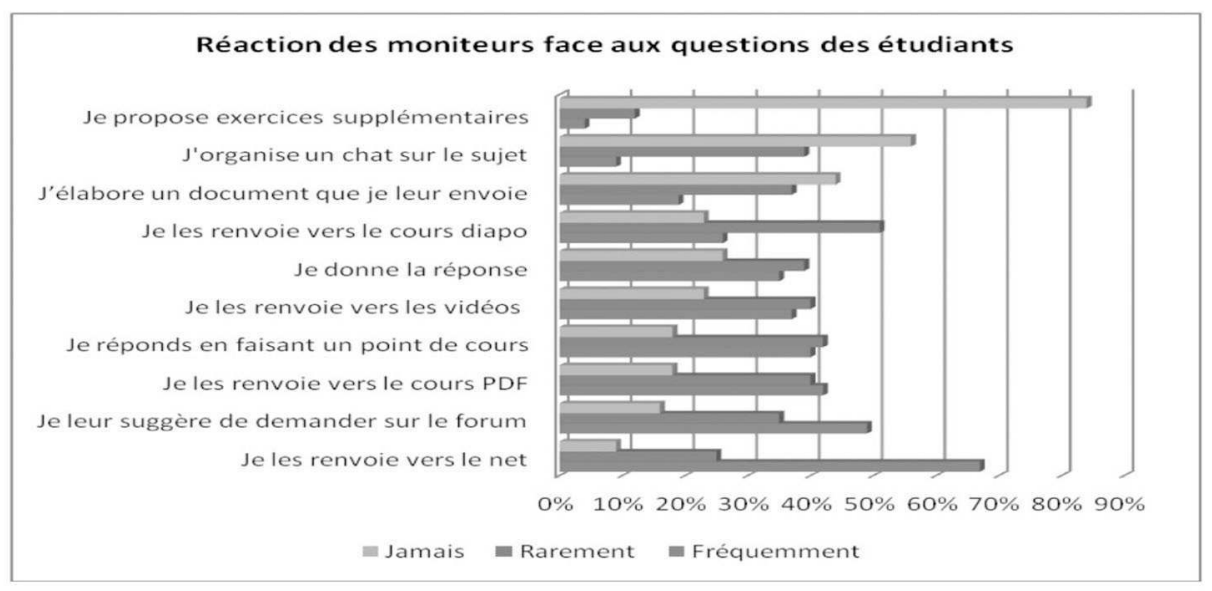

Figure 1 : Réactions des moniteurs face aux questions des étudiants

\section{Discussion : de l'intérêt des SHS}

Bien que présentée ici partiellement, cette recherche de compréhension des (non-)usages des ressources mises à disposition des apprenants préparant le C2i en formation hybride souligne la variété des facteurs à prendre en compte dans le cas de formation intégrant des TIC.

Si le fait de rencontrer des difficultés dans la préparation du C2i est corrélé à l'usage des contenus et possibilités d'interaction proposées, on constate que nombre d'étudiants en difficulté ne font pas ou peu usage des ressources mises à leur disposition, ce qui explique sans doute en partie l'importance des échecs à la certification. Alors que l'accès aux contenus et moyens de communication ne pose pas plus de problèmes en termes d'équipement que d'accessibilité technique, la compréhension de ce phénomène implique notamment la prise en compte de certains habitus, comme le soulèvent les exemples pris au niveau du travail scolaire et de la communication ainsi que l'appréhension des pratiques au regard du fonctionnement du dispositif de formation. Ces quelques résultats doivent évidemment être affinés au cours d'entretiens de recherche. Ils suffisent cependant, dès à présent, à souligner une fois encore l'intérêt des études en SHS dans ce domaine.

En effet, si la pertinence des recherches sur les EIAH s'intéressant à la modélisation de l'apprentissage, au développement et à l'évaluation de produits destinés à la transmission de savoirs ne peut être remise en cause tant les expérimentations mises en œuvre sont essentielles au développement d'environnements adaptés à la formation, cette orientation reste fortement techno-centrée en ce sens où l'apprenant est considéré comme un utilisateur quelque peu soumis à la machine. Or, si les dispositifs de formation sont conçus pour favoriser la formation et permettre le suivi de l'activité par différents éléments de tracking, l'orientation " utilisateur » omet parfois la complexité 
caractéristique de l'être humain. C'est ainsi que les SHS considèrent davantage ce dernier comme un usager dont les actions et pratiques doivent être étudiées en situation. Dès lors, à l'instar de Tchuentel et al. (2011) - mettant en avant que le recueil de données via les API (Application Programming Interface) est précieux et offre des pistes de compréhension des liens et centres d'intérêt des réseaux socionumériques, tout comme les recherches en sciences humaines qui permettent notamment de mieux comprendre les usages sociaux et les liens existants sur ces réseaux en prenant en compte ceux existants en dehors de ceux-ci - qui appellent à des recherches interdisciplinaires permettant de tenir compte de la double dimension technique et sociale de ces réseaux, ou de Marquet (2007) qui émet le souhait d'un meilleur équilibrage entre les STIC dominants actuellement dans les études d'EIAH- et les SHS, nous concluons cet article sur une invitation à l'ouverture favorisant la complémentarité des approches.

\section{BIBLIOGRAPHIE}

ALBERO B., THIBAULT F., « La recherche française en sciences humaines et sociales sur les technologies en éducation ", Revue française de pédagogie, 169, 2009, p. 53-66.

BOUTET A., TREMENBERT J., « Mieux comprendre les situations de non-usages des TIC. Le cas d'Internet et de l'informatique. Réflexions méthodologiques sur les indicateurs de l'exclusion dite numérique ", Les Cabiers du Numérique, 1, 2009, p. 69-100.

CAVIALE O., BRUILLARD E., « Les jeux d'acteurs sur des listes de discussion institutionnelles d'enseignants ", Réseaux, 155, 2009, p. 138-176.

CERTEAU de M., L'invention du quotidien, 1. Arts de faire, Paris, Gallimard, 1980-1990.

CHAPTAL A., "Usages prescrits ou annoncés, usages observés. Réflexions sur les usages du numérique par les enseignants », Document numérique, 3, 2007, p. 81-106.

GRANJON F., "Inégalités numériques et reconnaissance sociale. Des usages populaires de l'informatique connectée ", Les Cabiers du Numérique, 1, 2009, p. 19-44.

JAUREGUIBERRY F., PROULX S., Usages et enjeux des technologies de communication, Toulouse, Erès, 2011.

MARQUET P., « EIAH, un état de l'art en français », Distances et savoirs, 4, 2007, p. 587-591.

PAPI C., "Quels usages des technologies dans la reprise d'études à distance ? ", RIPES, 1, 2009, p. 62-79.

PAPI C. «Causes et motifs du non-usage de ressources numériques. Logiques d'usage des étudiants en formation initiale ", Recherches et Educations, à paraitre.

PERRIAULT J., La logique de l'usage, Paris, Gallimard, 1989. 
SMITH A., Home Broadband 2010. Pew internet \& American Life Project, [en ligne] http://www.pewinternet.org/Reports/2010/Home-Broadband-2010.aspx, 2010 (consulté le 10/02/2011).

TCHUENTE D. et al., « Pour une approche interdisciplinaire des TIC : Le cas des réseaux socionumériques ", Document numérique, 1, 2011, p. 31-57.

VON PAPE T., MARTIN C., « Non-usage du téléphone portable : au-delà d'une opposition binaire usagers/non-usagers ", Questions de communication, 18, 2010, p. 113-144.

WYATT S. "Les non-usagers de l'internet. Axes de recherche passés et futurs ", Questions de communication, 18, 2010, p. 21-36.

Résumé : Partant de la position intermédiaire des recherches sur les technologies de l'information et de la communication dans l'éducation (TICE) entre sciences et technologies de l'information et de la communication (STIC) et sciences humaines et sociales (SHS), nous mettons en avant la nécessaire complémentarité des différents champs de recherche et la pertinence de l'approche en termes d'usages sociaux, dès lors qu'il s'agit de saisir les pratiques mises en œuvre dans le cadre d'un dispositif de formation. Pour ce faire, nous prenons l'exemple de l'usage et du non-usage des ressources proposées aux étudiants dans le cadre de la préparation du $\mathrm{C} 2 \mathrm{i}$. Celui-ci fait effectivement ressortir la nécessité de ne pas limiter les TICE à des questions d'ordre technologique, voire cognitif, mais de prendre également en compte les dimensions pédagogiques et sociales susceptibles d'influencer usages et pratiques.

Mots-clés : C2i, EIAH, FOAD, usages, TIC.

Abstract : Based on the peculiar position of researches about information and communication technologies in education between information and communication technologies sciences and social and human sciences, we put forward both the benefits of researches about ICT uses in education and the complementarity of the different approaches. As an example we investigated about uses and nonuses of different online resources offered to students preparing the Internet and Computer Certificate and put into relief that technological and cognitive factors are not sufficient to explain uses and practices in an interactive learning environment. It seems indeed necessary not to forget the pedagogical and social dimensions at stake.

Keywords : Internet and Computer Certificate, ICT, ILE, ODL, Uses. 